\title{
Muscle fibre type composition, motoneuron firing properties, axonal conduction velocity and refractory period for foot extensor motor units in dystrophia myotonica
}

\author{
J BORG, ${ }^{*} \dagger$ L EDSTRÖM,* G S BUTLER-BROWNE, $\ddagger$ L-E THORNELL§ \\ From the Department of Neurology, ${ }^{*}$ Karolinska Hospital, and Söder Hospital, $\uparrow$ Stockholm, Sweden, \\ Department of Biologie Moléculaire, $\ddagger$ Institute Pasteur, Paris, France, and Department of Anatomy, $\S$ \\ University of Umeå, Sweden
}

SUMMARY Seven patients with dystrophia myotonica were investigated using neurophysiological combined with histochemical techniques to elucidate motor unit properties in foot extensor muscles, which are often involved in the early stages of this disorder. For the 25 extensor digitorum brevis motor units studied the axonal conduction velocity, the axonal refractory period and the voluntary firing properties were within normal limits. However, high threshold motor units were not observed and the mean value of the axonal conduction velocities was lower $(p<0.02)$ for the dystrophia myotonica motor units when compared with corresponding data from healthy subjects. There were also signs of impaired impulse propagation in the terminal part of the motor unit. In muscle biopsy specimens from the anterior tibial muscle, fibre type composition and structure were demonstrated using enzyme histochemical techniques for adenosine-triphosphatase and immunohistochemical techniques for identification of the types of myosin isoform present. The histochemical findings indicated a type I fibre dominance, which was most obvious in the more seriously affected muscles. Neonatal myosin was observed preferentially in small but also in some normal sized fibres. Furthermore, some ring fibres were present and these showed staining with antineonatal myosin in their superficial portion. This indicates that an abnormal regeneration is one cause of the myopathic appearance of the muscle fibres in dystrophia myotonica. These investigations show that there is a reduced proportion of type II motor units in foot extensor muscles involved in the myopathy in dystrophia myotonica although it cannot definitely be established whether this is due to a loss of high threshold type II motor units or type II to type I transformation.

One of the earliest and most prominent features of muscle biopsies from patients with dystrophia myotonica is a selective atrophy of slow twitch fibres (type I). This was initially demonstrated by enzyme histochemical ATPase techniques ${ }^{1}$ and further confirmed by immunohistochemical methods for fast and slow tropomyosin. ${ }^{2}$ The proportion of muscle fibre types present in different muscles in dystrophia myotonica has however been less well documented. According to Radu et al $^{\mathbf{3}}$ a type I fibre dominance is

Address for reprint requests: Dr J Borg, Department of Neurology, Karolinska Hospital, S-10401 Stockholm, Sweden.

Received 11 November 1986 and in revised form 28 January 1987. Accepted 30 January 1987 typical for dystrophia myotonica. Similar findings were also reported for congenital dystrophia myotonica. ${ }^{4}$ Farkas et al ${ }^{5}$ have, however, reported a predominance of type II fibres (fast twitch type) in cases of congenital dystrophia myotonica investigated in the immediate postnatal stage. Most earlier investigations were, however, performed on proximal extremity muscles and the discrepancies between the reports of fibre type composition might be the results of investigating different muscles at different ages and different stages of the disease. A type I fibre dominance in the foot extensors is in accordance with the studies carried out by McComas et $\mathrm{al}^{6}$ on motor unit properties in extensor digitorum brevis in dystrophia myotonica but does not accord with studies of 
Belanger and $\mathrm{McComas}^{7}$ on the contraction time of extensors and plantar flexors of the foot in dystrophia myotonica. In conclusion, there is no evidence that the early type I fibre atrophy reported in some muscles indicate a general susceptibility in that type of fibre resulting in a successive loss of slow muscle fibres during the course of the disease, especially since more recent studies show in fact a predominance of type I fibres in dystrophia myotonica.

The early and striking involvement of distal muscles in dystrophia myotonica suggests that disturbed innervation could play a role in the pathogenesis of the disease. This was originally suggested by Coers and Woolf ${ }^{8}$ and MacDermot ${ }^{9}$ on the basis of studies carried out on distal nerves and nerve terminals. The question as to whether muscle affection in dystrophia myotonica is primarily of muscular or neurogenic origin cannot definitely be answered.

In the present study of patients with dystrophia myotonica, muscle biopsy material from a distal muscle (anterior tibial muscle) was investigated with regard to muscle fibre type composition and fibre characteristics by ATPase enzyme histochemistry and isomyosin immunohistochemistry. The muscle biopsy findings were correlated with the axonal conduction velocity, the axonal refractory period and the voluntary properties of single motor units.

\section{Material and methods}

The study was carried out on two women and five men, aged 35-46 years, with clinically and neurophysiologically typical dystrophia myotonica. All patients had impaired force of foot dorsiflexion (Kendal 2-4).

The electrophysiological study was performed in m extensor digitorum brevis (EDB). Electromyographic recordings were made by conventional, bipolar needle electrodes (DISA 9013K0802, Electronic, Skovlunde, Denmark). Single motor unit recordings permitting the identification of the test motor unit potential even at maximal voluntary contraction and after supramaximal nerve stimulation by electric shocks were possible owing to a reduced number of motor units ${ }^{6}$ and increased motor unit fibre density. ${ }^{10}$ After identification of the test motor unit potential during sustained voluntary contraction the voluntary effort was reduced until the test motor unit ceased to fire and electrical stimuli were delivered. The test potential was characterised by its size and shape and all-or-none appearance.

The common peroneal nerve was stimulated proximally at the fibular head and distally at the ankle. Stimuli were delivered through surface electrodes $0.6 \mathrm{~cm}$ in diameter. The cathode was placed over the nerve and the anode $2-3 \mathrm{~cm}$ laterally or medially to the cathode. Rectangular pulse waves of $0.2 \mathrm{~ms}$ duration were used. Stimulus strength could be gradually changed from $0-100 \mathrm{~mA}$. Electrical stimuli were delivered as follows:

1. A single nerve stimulus was delivered proximally and distally, the latency difference of the motor unit responses was calculated and the axonal conduction velocity determined.
2. A single proximal and a single distal stimulus were then delivered coupled to each other. Stimulus strength was $10 \%$ above the axonal threshold at rest. The proximal stimulus was initially delayed $10-15 \mathrm{~ms}$ to permit the distally evoked, antidromic propagated nerve impulse to pass the proximal test point before stimulation. The stimulus intervals were then reduced by $0.2 \mathrm{~ms}$ intervals until the second evoked motor unit potential disappeared. This was due to blocking at the proximal testing point during the refractory period after the antidromic impulse. By comparing the shortest stimulus interval without blocking with the previously determined latency difference (point 1 above) the axonal refractory period following an antidromic propagated nerve impulse was calculated. ${ }^{11}$
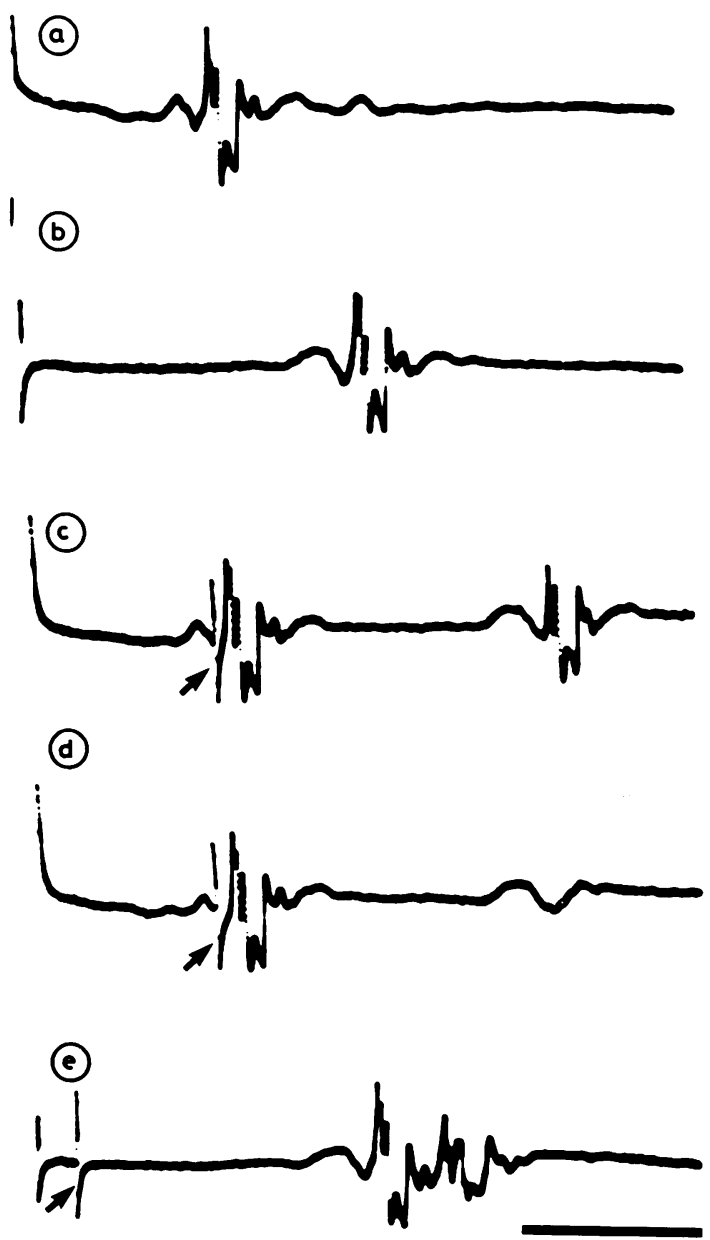

Fig 1 Electromyographic single motor unit recordings after delivering $(A)$ one distal nerve stimulus, $(B)$ one proximal nerve stimulus, $(C)$ one distal and one proximal nerve stimulus coupled to each other with the proximal stimulus delayed $10.5 \mathrm{~ms}$ and $(D)$ with the proximal stimulus delayed $10 \cdot 3 \mathrm{~ms}$ and $(E)$ paired proximal stimuli with $2.5 \mathrm{~ms}$ stimulus interval. Arrow's indicate delayed stimulus. Time bar $10 \mathrm{~ms}$. 


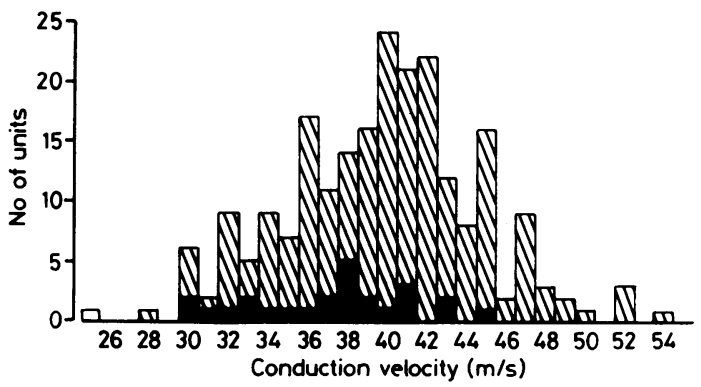

Fig 2 Axonal conduction velocity spectrum in normal subjects (shaded) and dystrophia myotonica patients (black).

3. Paired electrical stimuli were delivered proximally with stimulus intervals of $3 \mathrm{~ms}$ or less. The motor unit response interval was determined. ${ }^{12}$

The minimal and maximal firing rates during sustained, voluntary contraction were defined for the test motor unit (cf Results).

Room temperature was $23-25^{\circ} \mathrm{C}$. Skin temperature was continuously controlled and held at $32^{\circ} \mathrm{C}$ by a DISA heater (DISA Electronic, Skovlunde, Denmark).

Muscle biopsies were obtained from the anterior tibial muscle using a percutaneous conchotome technique. The biopsies yielded at least two specimens from each patient and most material was immediately frozen in liquid Freon 22 kept at its melting point by liquid nitrogen. Frozen sections $10-16 \mu \mathrm{m}$ thick were cut in a cryostat. The sections were stained for demonstration of myofibrillar ATPase at $\mathrm{pH}$ $9 \cdot 4^{13}$ and after preincubation at $\mathrm{pH} 4 \cdot 3,4 \cdot 6$ and $10 \cdot 3^{14}$ with haematoxylin and eosin and modified trichrome stains. For details regarding the methods see Dubowitz and Brooke. ${ }^{15}$ Sections in parallel with those produced for enzyme histochemistry were processed for immunohistochemical demonstration of myosin types.

Polyclonal rabbit antibodies made against neonatal, adult fast and adult slow rat myosin heavy chain were rendered specific by crossabsorption as described previously. ${ }^{16} 17$ These antibodies to rat myosins cross-react with the heavy chains of human myosins as shown by immunotransfer reactions performed on extracts of human muscle biopsies (Butler-Browne and Whalen, unpublished results). The antibodies were used both with the indirect peroxidase and antiperoxidase (PAP) stained essentially according to Billeter et $a l^{18}$ and in indirect immunofluorescence essentially as described by Butler-Browne et al. ${ }^{16}$ The FITC conjugated goat antirabbit Ig and the PAP reagents were purchased from Dakopatts, Denmark. The sections for immunofluorescence were mounted in Mowiol (Hoechst, Frankfurt, West Germany) and were viewed in a Leitz Wetzlar Orthoplane microscope equipped with epifluorescence optics and a fluorescein and a rhodamine filter pack. The PAP stained sections were viewed with a Leitz Dialux microscope.

\section{Results}

Axonal conduction velocity and refractory period The axonal conduction velocity and refractory period were determined for $25 \mathrm{EDB}$ motor units. One to six motor units were studied in each of the seven patients. Figure 1A-D illustrates the recordings when the axonal conduction velocity and refractory period were determined. In $\mathrm{A}$ the test motor unit potential was recorded after one distal stimulus and in B after one proximal stimulus. The latency difference was $8.3 \mathrm{~ms}$ which for this motor unit corresponded to a conduction velocity of $37 \mathrm{~m} / \mathrm{s}$. In $\mathrm{C}$ the test potential was recorded when the distal stimulus preceded the proximal stimulus by $10.5 \mathrm{~ms}$. At this long stimulus interval the proximal stimulus elicited a response. In $\mathrm{D}$, when the stimulus interval was reduced to $10.3 \mathrm{~ms}$, blocking occurred at the proximal stimulus point due to refractoriness after the antidromic propagated impulse from the ankle and the proximal stimulus failed to elicit a response. Thus, for this motor unit the axonal refractory period after an antidromic propagated impulse was $2 \cdot 2 \mathrm{~ms}(10 \cdot 5-8 \cdot 3)$.

Figure 2 shows the axonal conduction velocities of the 25 motor units together with corresponding data from 222 motor units in healthy subjects. ${ }^{19}$ In dystrophia myotonica the axonal conduction velocity range was $30-45 \mathrm{~m} / \mathrm{s}$ as compared with $25-54 \mathrm{~m} / \mathrm{s}$ in healthy subjects. The mean value of the conduction velocities for all dystrophia myotonica motor units was $37 \cdot 20 \pm 4 \cdot 13(\mathrm{M} \pm \mathrm{SD})$. This was significantly lower $(p<0.02)$ than corresponding value for the 222 motor units in healthy subjects $(39 \cdot 75 \pm 4 \cdot 86)$. Except for a reduced proportion of fast conducting motor units in dystrophia myotonica the conduction velocity distributions corresponded well.

The axonal refractory period after a conditioning antidromic propagated impulse ranged from 1.4 to $3.2 \mathrm{~ms}$. Except for two motor units in one patient with refractory periods longer than $2.5 \mathrm{~ms}$ the refractory periods were within the range observed in healthy subjects. ${ }^{20}$

Figure 3 shows the individual mean values of the axonal conduction velocities and refractory periods

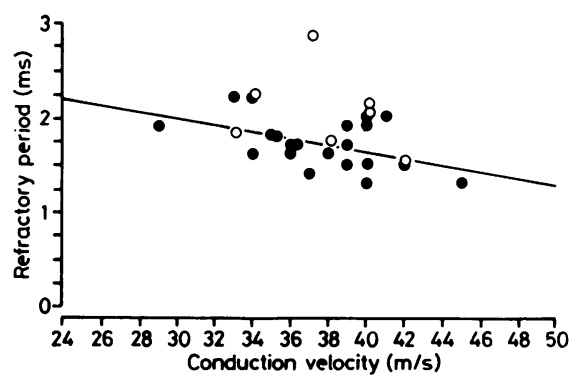

Fig 3 Axonal conduction velocity and refractory period for 22 healthy subjects (filled dots) and 7 dystrophia myotonica patients (unfilled dots). Regression line for data from healthy subjects marked as illustration. 


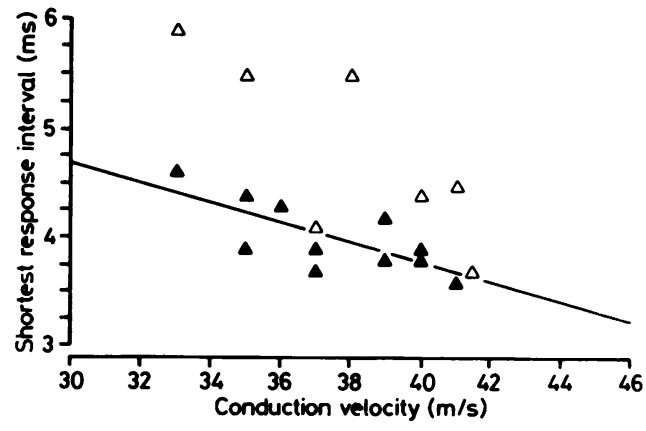

Fig 4 Shortest motor unit response interval after delivering paired electrical stimuli proximally. Mean values for 11 healthy subjects (filled triangles) and 7 dystrophia myotonica patients (unfilled triangles). Regression line for dat a from healthy subjects marked as illustration.

of the seven patients together with corresponding data from normal subjects. ${ }^{20}$ Considering the inverse relationship between the axonal conduction velocity and refractory period ${ }^{11}$ and that the conduction velocities were higher in healthy subjects, the mean value of the refractory periods, $2.02 \pm 0.39 \mathrm{~ms}$ was not significantly different $(p>0.05$ ) from the corresponding value in normal subjects $(1 \cdot 76 \pm 0 \cdot 26)$.

\section{Shortest motor unit response interval}

When paired electrical stimuli were delivered proximally to the peroneal nerve at intervals shorter than $3 \mathrm{~ms}$, the slowing of the conduction of the second impulse propagated during the relative refractory period of the first impulse normally prevented peripheral blockings and limited the motor unit response interval to $3-5 \mathrm{~ms}^{12}$

For the 25 motor units paired electrical stimuli were delivered proximally to the peroneal nerve at stimulus intervals shorter than $3 \mathrm{~ms}$. For $68 \%(17 / 25)$, of the motor units, having axonal conduction velocities $32-45 \mathrm{~ms}$, the shortest motor unit response interval ranged from 3.3 to $7.9 \mathrm{~ms}$. For three motor units it was longer than $5 \mathrm{~ms}$ which was never observed in healthy subjects. ${ }^{12}$

Figure 4 shows the individual mean values of the shortest motor unit response intervals and axonal conduction velocities for the seven patients together with corresponding data from healthy subjects. ${ }^{12}$ The mean value of the shortest response interval in the patients, $4.80 \pm 0.83 \mathrm{~ms}$, was significantly longer $(p$ $<0.02$ ) than corresponding values in healthy subjects $(4.06 \pm 0.30)$.

For $32 \%(8 / 25)$ of the motor units significant peripheral blocking occurred when the stimulus interval was less than $3 \mathrm{~ms}$ as illustrated in fig $1 \mathrm{E}$. When paired electrical stimuli were delivered at the proximal stimulus point with $2.5 \mathrm{~ms}$ intervals the second motor unit potential was significantly reduced and the potential identity was lost. This was not seen in healthy subjects. ${ }^{12}$

\section{Voluntary firing properties}

For 15 of the 25 EDB motor units the voluntary firing properties were studied during voluntary sustained contraction and defined according to the same criteria as used in previous studies. ${ }^{2122}$ For the other 10 motor units the identity of the recorded potential was lost due to displacement of the needle electrode or due to myotonic discharges.

Six motor units were low threshold, tonically firing motor units with minimum firing rate below $10 / \mathrm{s}$ and maximum firing rate about $30 / \mathrm{s}$. They had axonal conduction velocities $30-41 \mathrm{~m} / \mathrm{s}$. Nine motor units were tonically firing motor units with minimum firing rate $10-20 / \mathrm{s}$ and maximum rate above $30 / \mathrm{s}$. They had axonal conduction velocities $38-45 \mathrm{~m} / \mathrm{s}$.

For the 15 motor units the voluntary firing properties and the relation between the firing properties and the axonal conduction velocity was the same as for corresponding motor units in normal subjects. ${ }^{21}$ However, in the EDB of healthy subjects there are also high threshold motor units with phasic firing pattern and axonal conduction velocities above $40 \mathrm{~m} / \mathrm{s}$. No motor unit of this type was identified in the patients (cf Discussion).

\section{Muscle biopsy findings}

All biopsies showed pathological changes. In the two least affected muscles the only aberration from normal was a high incidence of multiple central nuclei and occasional atrophic fibres. In highly affected muscles there was a variety of pathological changes which conformed to well known characteristics of dystrophia myotonica muscle: ring-fibres with and without sarcoplasmic masses, multiple central nuclei, fibre splitting, occasional necrotic fibres, cytoplasmic bodies and increased amount of connective tissue. Muscle fibre type characteristics In slightly affected muscles there was a clear differentiation between type I and type II muscle fibres as demonstrated histochemically with the ATPase technique (fig 5A, B). Type I fibres dominated and some scattered fibres were atrophic. Most type II fibres were of the type IIA according to the terminology of Brooke and Kaiser. ${ }^{14}$

In more advanced myopathic muscle the type IIA fibres were scarce or even absent resulting in a homogeneous population of fibres with almost identical staining characteristics for myofibrillar ATPase (fig 5C, D, E). These fibres exhibited a high content of acid stable ATPase (demonstrated at $\mathrm{pH} 4.3$ and 4.6) but also a higher content of alkali stable ATPase than in ordinary type I fibres as demonstrated by the original method of Padykula and Herman ${ }^{13}$ without pre- 


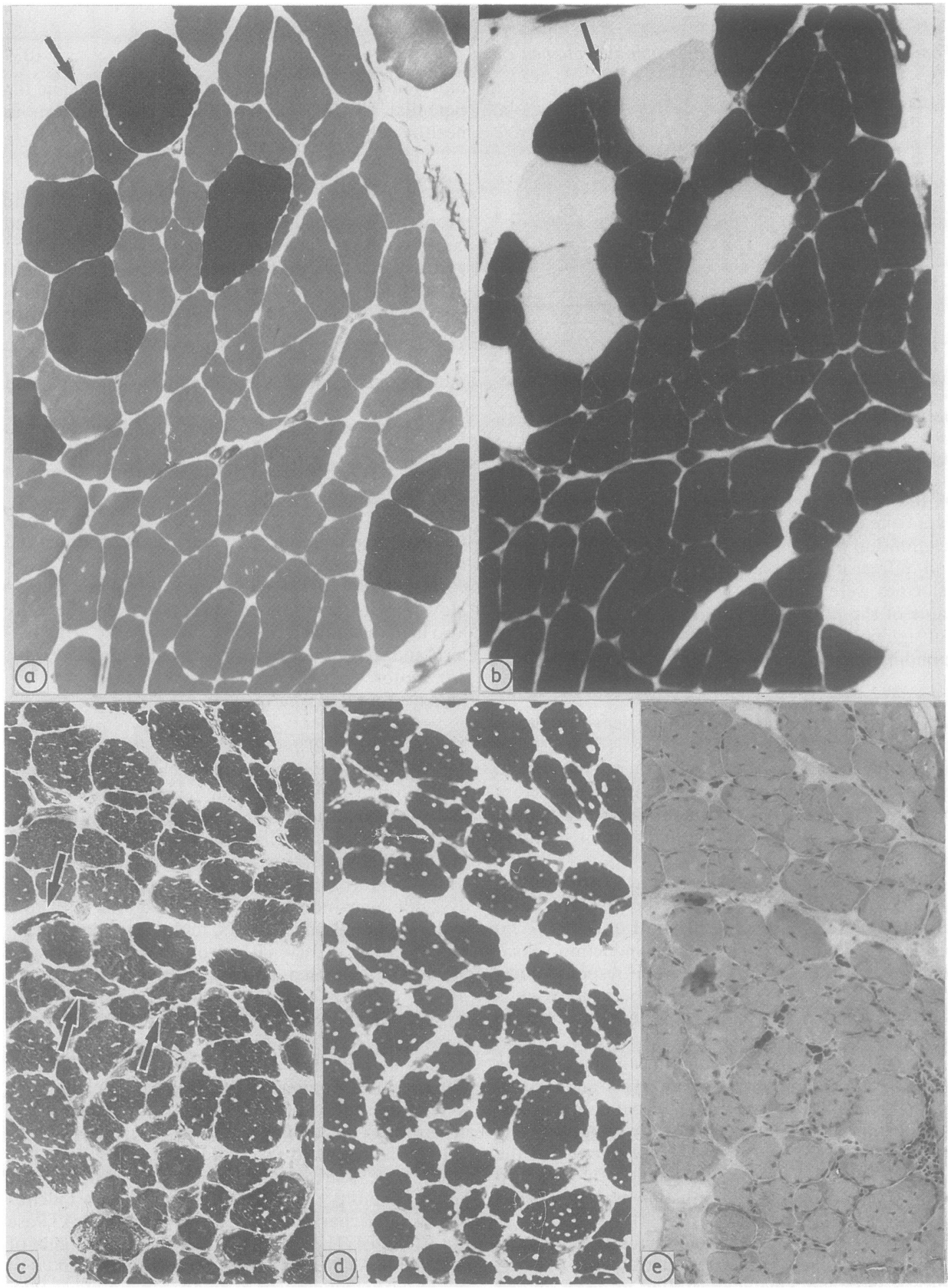

Fig 5 Transverse sections of anterior tibial (A) muscle fibres in dystrophia myotonica. A and B represent a moderately affected muscle stained for myofibrillar ATPase at $\mathrm{pH} 9.4$ without preincubation and after preincubation at pH 4.6 respectively. The majority of fibres are of type I and lightly stained in $A$ and darkly in $B$ but there are some type IIA fibres visible as well, darkly stained in $A$ and lightly in $B$. Note the occasional intermediately stained fibres with high stainability in both $A$ and $B$ (one fibre is indicated by an arrow). C, D and $E$ are a series of transverse sections from a severely affected muscle (AT). Only slight variation in ATPase activity is seen when stained for ATPase at pH $9 \cdot 4$ without acid preincubation. Small fibres indicated by arrows show high activity $(C)$. After preincubation at pH 4.3 (D) almost all fibres show high enzyme activity. Note numerous myopathic changes as seen after staining with haematoxylin and eosin $(E) . A-B \times 125 ; C-E \times 100$. 

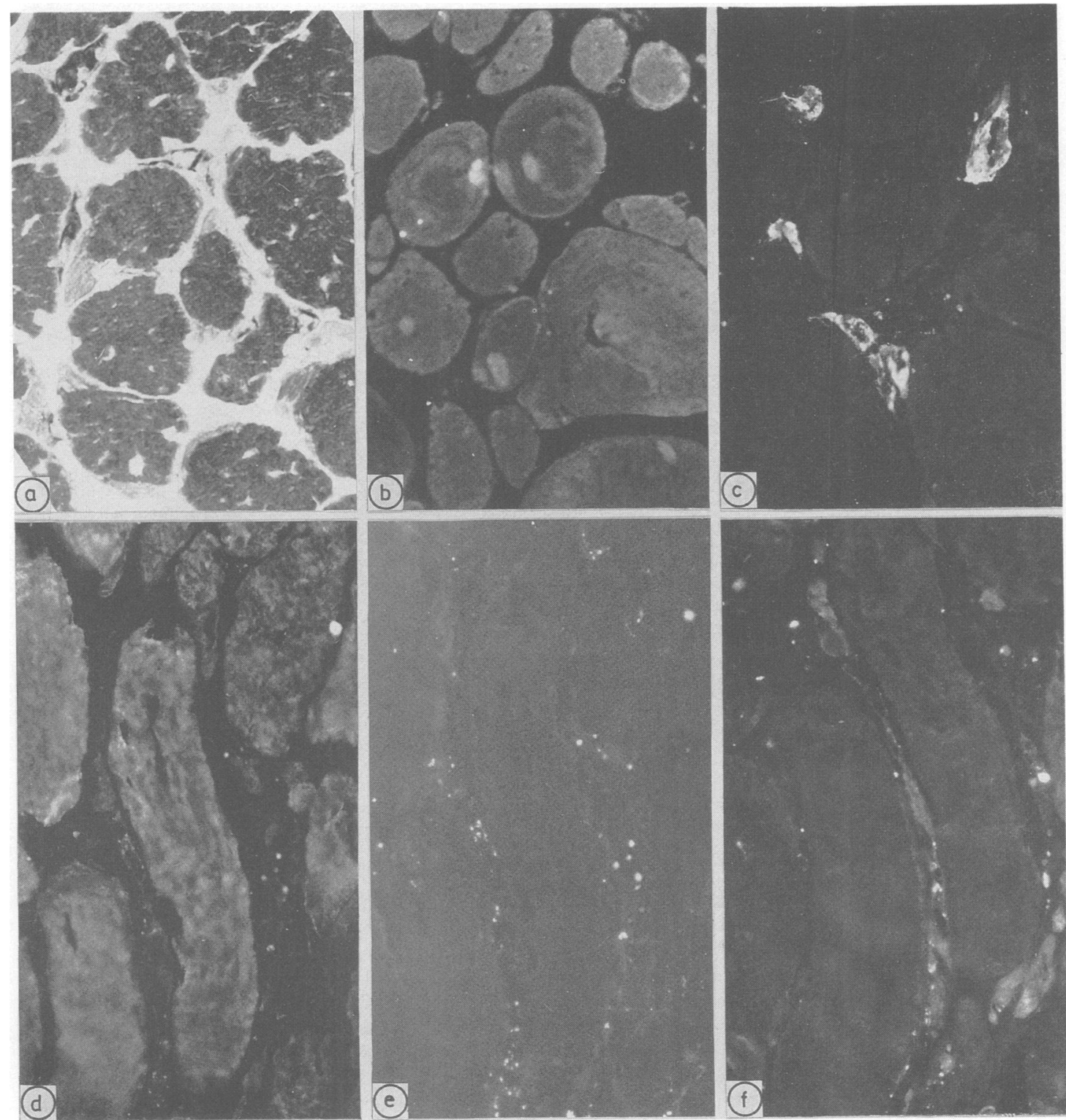

Fig $6 A, B$ and $C$ are sections from the same specimen as $5 C-E$ but at a higher magnification. In $A$, stained for myosin ATPase at pH 9.4 without preincubation, small fibres with higher ATPase activity (one indicated by an arrow) contrast with the surrounding larger intermediately stained fibres. In sections labelled with anti-slow myosin almost all fibres show high degrees of staining $(B)$. A few small fibres are labelled with antibodies against antineonatal myosin $(C)$. Longitudinal sections are stained with anti-slow myosin $(D)$ and anti-fast myosin $(F)$. Note that all fibres are stained in $(D)$ and unstained in $(F)$. $E$ is a control and show's the same area of the section in $D$ but viewed at another wave length to reveal autofluorescence of lipofuscin granules. $A \times 250 ; B \times 125 ; C-F \times 310$. 


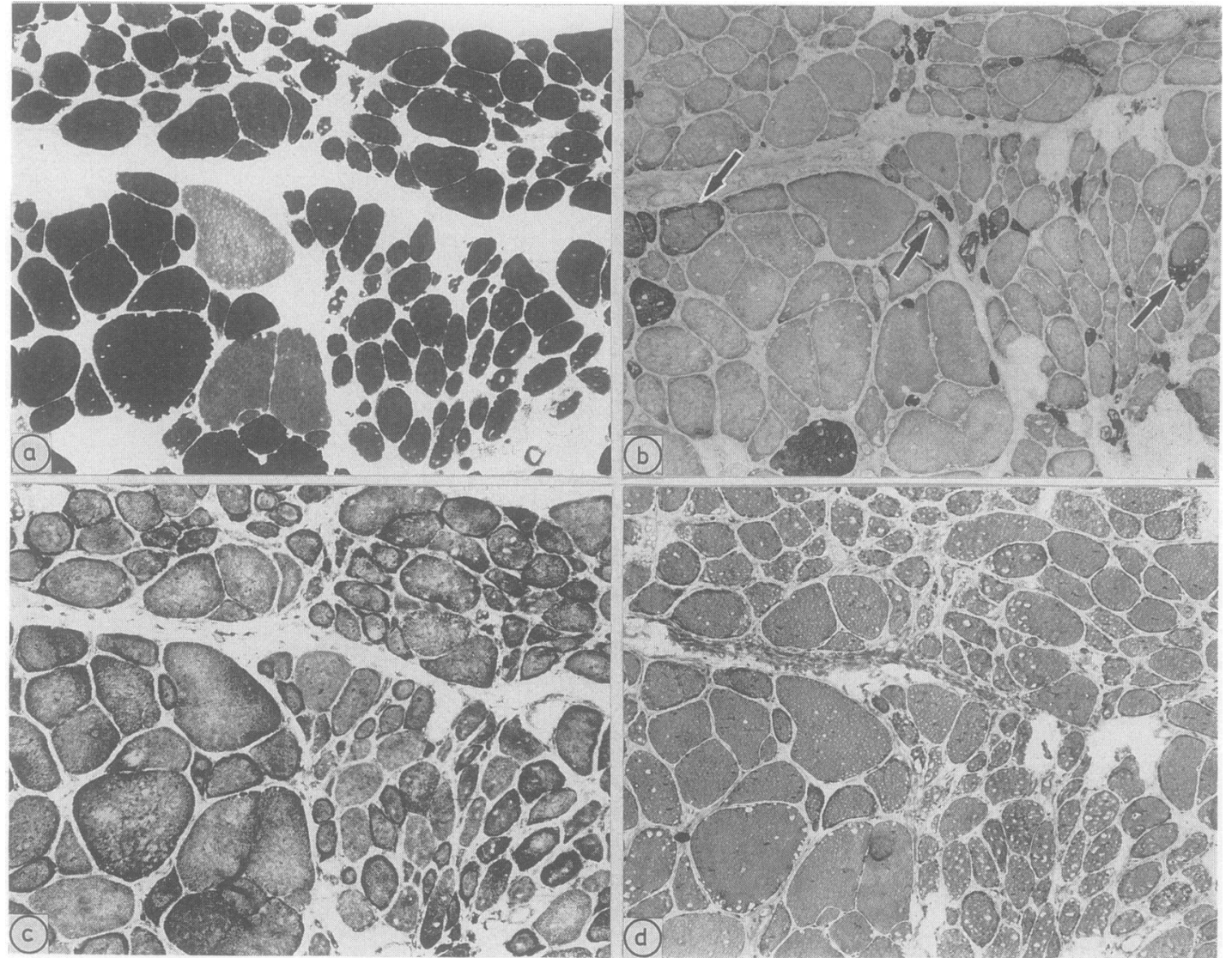

Fig 7 Serial cross sections of a severely affected muscle $(A T)$ stained for $(A)$ ATPase at pH 4.6, (B) antineonatal myosin visualised with the PAP method, (C) NADH-TR and (D) Gomori trichrome. Many small fibres are labelled with antineonatal myosin. Note also that three ring fibres (arrows) are mainly labelled in their outer portion with antineonatal myosin. $\times 100$.

incubation and thus the staining characteristics were similar to fibres of type IIC according to the terminology of Brooke and Kaiser. ${ }^{14}$ When stained for ATPase with an akali preincubation at $\mathrm{pH} 10.3$ most fibres, however, were weakly stained or unstained which suggested that they had type I characteristics.

The immunohistochemical analyses conformed with the enzyme histochemical concept that most fibres in the muscle with lack of fibre differentiation exhibited a high content of slow myosin and very few fibres contained significant levels of fast myosin (fig 6F). Furthermore, some fibres consistently contained neonatal myosin (figs 6C and 7B). Of special interest was the observation that ring fibres contained neonatal myosin in the outer superficial portion of the fibres (fig 7).

\section{Discussion}

This study of foot extensors in dystrophia myotonica has shown that there is a successive disappearance of type II muscle fibres during the course of the disease as demonstrated in muscle biopsies and a parallel disappearance of high threshold, fast conducting motoneurons indicating a successive loss or transformation of type II motor units as a result of the disorder. The residual muscle fibre population had the characteristics of a type I fibre with reference to immunohistochemically demonstrated slow isomyosin content and might then be organised in slow twitch motor units. It must, however, be emphasised that these muscle fibres are not normal type I fibres either structurally or with reference to enzyme histochemical fibre typing based on staining for myosin ATPase, and thus their contraction properties and innervation cannot unreserved be integrated in a concept for motor unit properties deduced from normal physiology and histochemistry.

The muscle fibre type characteristics of AT in dys- 
trophia myotonica are strikingly similar to those in biopsies from AT in the distal myopathy of Welander. ${ }^{2324}$ In Welander's disease there is also an early but slight type I fibre atrophy and later during the course of the disease there is a successive loss of type II fibres and the residual fibres have type characteristics partly of intermediate character ${ }^{23}$ and partly of type I. ${ }^{24}$ Thus, it is obvious that the type I fibre atrophy reported as typical for muscles in dystrophia myotonica is not ultimately followed by a successive disappearance of type I muscle fibres or type I motor units, at least in the foot extensors which are early and often highly involved in dystrophia myotonica. On the contrary, there is a clear indication of disappearance of normal type II motor units. Thus the pathophysiological significance of early type I fibre atrophy is obscure in dystrophia myotonica as well as in Welander's disease. Another similarity between dystrophia myotonica and Welander's disease was the presence of neonatal myosin in some fibres. From experimental studies it is known that during muscle development and muscle regeneration myosin isoforms appear, in a sequential manner: EmbryonicNeonatal-(Fast)-Slow. ${ }^{25-27}$ Our observations of ring fibres containing neonatal myosin in the outer portion of the fibres would indicate a process of unsuccessful regeneration. This observation supports the idea that ring fibres are formed by activated satellite cells which differentiate but fuse with the material in an abnormal way. ${ }^{28}$ As most ring fibres did not show staining for neonatal myosin it is likely that those which did were in the early stages of ring fibre formation. However, it must be emphasised that the other structural and ultrastructural changes of muscle fibres are strikingly different in Welander's disease ${ }^{24}$ as compared with dystrophia myotonica, ${ }^{29}$ hinting at a different basic pathogenetic mechanism. Nevertheless, there might be similarities with reference to motoneuron disturbance. A neurogenic factor has been proposed as a basis for some of the changes in Welander's disease. ${ }^{22}$ However, the abnormal motoneuron firing pattern demonstrated in Welander's disease was not seen in dystrophia myotonica motor units.

It may also be speculated whether in the paretic AT muscle, an abnormal use of motor units in locomotion, as has been described in peroneal palsy by Grimby et al ${ }^{30}$ induces some muscle fibre transformation of the same type as known from animal experiments demonstrating fibre transformation after longterm electrical stimulation (for review cf Edström and Grimby ${ }^{31}$ ). Recent observations in muscles subjected to abnormal overuse due to reduction of normal TA muscle power after poliomyelitis suggest that such a mechanism of fibre transformation is operating also in man at least during pathological condi- tions. $^{32}{ }^{33}$ Similarly, recent observations of a high percentage of fibres with promiscuous expression of myosin light chains in dystrophia myotonica ${ }^{34}$ would be compatible with an altered motoneuron firing.

The suggestion of a neurogenic factor operating in dystrophia myotonica ${ }^{89}$ has been subject to criticism. It has been doubted as to whether the early changes in distal nerve twigs and nerve terminals are of primary neurogenic origin. ${ }^{2935} 36$ Drachman and Fambrough $^{37}$ failed to demonstrate increased extrajunctional ACh-receptor sites even in atrophic muscle fibres although such increase in receptor sites were easily demonstrated in motoneuron disease.

In our recordings from the EDB there was an abnormal delay of the second motor unit response and peripheral blockings when paired electrical stimuli were delivered proximally to the peroneal nerve. An abnormally long delay would be expected if the relative refractory period of the nerve fibres was increased. ${ }^{12}$ Since the refractory period as measured proximally was within the same range as in healthy subjects the present abnormalities are due to impaired conduction and safety factor, either in the terminal nerve, the neuromuscular transmission or the muscle fibres. It is well known that there is a decline of the muscle action potential at low frequency repetitive nerve stimulation in myotonic disorder. ${ }^{638}$ The reason for this is not known. In myotonic dystrophy there are morphological abnormalities in the nerve terminal as described by Coers $\mathrm{et}^{\mathbf{8}} \mathrm{l}^{\mathbf{2}}$ and it seems probable that impaired conduction and safety factor at this level contribute to the present findings of an abnormal delay and blockings.

Our data on the axonal conduction velocities and refractory periods as measured in the proximal axon segment were within normal ranges as were the voluntary firing properties. Thus, with regard to these parameters, no signs of generalised motoneuron disturbance were present. However, a reduced proportion of high threshold motor units with high axonal conduction velocities was found in dystrophia myotonica as compared with healthy subjects. These findings are in agreement with previous reports of loss of motor units. ${ }^{6}$ However, Pollock and Dyck ${ }^{39}$ failed to demonstrate signs of nerve fibre loss or ultrastructural nerve fibre changes in dystrophia myotonica.

This study was supported by grants from the Swedish Medical Research Council (14X-4749, 12X-3875, $12 \mathrm{X}-3934)$ and the Vivian Smith Foundation.

\section{References}

1 Engel WK, Brooke MH. Histochemistry of the myotonic disorders. In: Kuhn E, ed. Progressive Muskeldystrophie, Myotonie, Myastenie. Heidelberg: Springer, 1966:203-22. 
2 Dhoot GK, Pearce GW. Transformation of fibre types in muscular dystrophies. J Neurol Sci 1984;65:17-28.

3 Radu H, Radu A, Blücher G. Quantitative study of the myotonic state. Correlative biochemical, histoenzymological and electrical investigations. Eur Neurol 1970;4:100-7.

4 Argov Z, Gardner-Medwin D, Johnson MA, Mastaglia FL. Congenital myotonic dystrophy. Fiber type abnormalities in two cases. Arch Neurol 1980;37:693-6.

5 Farkas E, Tomé FMS, Fardeau M, Arsenio-Nunes ML, Dreyfus P, Diebler MF. Histochemical and ultrastructural study of muscle biopsies in 3 cases of dystrophia myotonica in the newborn child. J Neurol Sci 1974;21:273-88.

6 McComas AJ, Campbell MJ, Sica REP. Electrophysiological study of dystrophia myotonica. J Neurol Neurosurg Psychiatry 1971;34:132-9.

7 Belanger AY, McComas AJ. Contractile properties of muscles in myotonic dystrophy. J Neurol Neurosurg Psychiatry 1983;46: 625-31.

8 Coers C, Woolf AL. The Innervation of Muscle: A Biopsy Study. Oxford: Blackwell Scientific Publications, 1959.

9 MacDermot $\mathrm{V}$. The histology of the neuromuscular junction in dystrophia myotonica. Brain 1961;84:75-84.

10 Stålberg E, Trontelj JV. Single Fibre Electromyography. Old Woking, Surrey: The Mirvelle Press Limited, 1979:134.

11 Borg J. Axonal refractory period of single short toe extensor motor units in man. J Neurol Neurosurg Psychiatry 1980;43: 917-24.

12 Borg J. Effects of prior activity on the conduction in single motor units in man. J Neurol Neurosurg Psychiatry 1983;46:317-21.

13 Padykula HA, Herman E. Factors affecting the activity of adenosine triphosphatase and other phosphatases as measured by histochemical techniques. J Histochem Cytochem 1955;3: 161-7.

14 Brooke MH, Kaiser KK. Muscle fibre types. How many and what kind? Arch Neurol 1970;23:369-79.

15 Dubowitz V, Brooke MH. Muscle Biopsy: A Modern Approach. London: WB Saunders, 1973.

16 Butler-Browne GS, Bugaisky LB, Cuenoud S, Schwartz K Denervation of newborn rat muscles does not block the appearance of adult fast myosin heavy chain. Nature 1982;299. $830-3$.

17 Butler-Browne GS, Whalen RG. Myosin isozyme transitions occurring during the postnatal development of the rat soleus muscle. Dev Biol 1984;102:324-34.

18 Billeter R, Weber H, Lutz H, Howald H, Eppenberger HM, Jenny E. Myosin types in human skeletal muscle fibers. Histochemistry 1980;65:249-59.

19 Borg J. Refractory period and conduction velocity of single motor nerve fibres in motoneuron disease. $J$ Neurol Neurosurg Psychiatry 1984;47:349-55.

20 Borg J. Refractory period of single motor nerve fibres in man. J Neurol Neurosurg Psychiatry 1984;47:344-8.

21 Borg J, Grimby L, Hannerz J. Axonal conduction velocity and voluntary discharge properties of individual short toe extensor motor units in man. J Physiol (London) 1978;277:143-52.

22 Borg J, Grimby L, Hannerz J. Motor neuron firing range, axonal conduction velocity and muscle fibre histochemistry in neuromuscular diseases. Muscle Nerve 1979;2:423-30.

23 Edström L. Histochemical and histopathological changes in skeletal muscle in late-onset hereditary distal myopathy (Welander). J Neurol Sci 1975;26:147-57.

24 Thornell LE, Edström L, Billiter R, Butler-Browne GS, Kjörell U, Whalen RG. Muscle fibre type composition in distal myopathy (Welander). J Neurol Sci 1984;65:269-92.

25 Whalen RG, Sell SM, Butler-Browne GS, Schwartz K, Bouveret $P$, Pinset-Hänström I. Three myosin heavy-chain isozymes appear sequentially in rat muscle development. Nature (London) 1981;292:805-9.

26 Fitzsimons RB, Hoh JFY. Embryonic and foetal myosins in human skeletal muscle-The presence of foetal myosins in Duchenne dystrophy and infantile spinal muscular atrophy. J Neurol Sci 1981;52:367-84.

27 Sartore S, Gorza L, Schiaffino S. Fetal myosin heavy chains in regenerating muscle. Nature 1982;298:294-6.

28 Chou SM, Nonaka I. Satellite cells and muscle regeneration in diseased human skeletal muscles. J Neurol Sci 1977;34:131-45.

29 Klinkerfuss GH. An electron microscopic study of myotonic dystrophy. Arch Neurol 1967;16:181-93.

30 Grimby L, Holm K, Sjöström L. Abnormal use of remaining motor units during locomotion in peroneal palsy. Muscle Nerve 1984;7:327-31.

31 Edström L, Grimby L. Effect of exercise on the motor unit. Muscle Nerve 1986;9:104-26.

32 Edström L, Borg J, Borg K, Grimby L. Excessive usr of remaining muscle fibres and selective loss of type II in antecedent polio. Muscle Nerve 1986;9:249.

33 Grimby L, Borg J, Borg K, Sjöström L. Excessive single motor unit discharge during walking in neuromuscular disorders. Muscle Nerve 1986;9:250.

34 Moore GE, Roses AD, Pericak-Vance MA, Garrett WE Jr, Schachat FH. Promiscuous expression of myosin in myotonic dystrophy. Muscle Nerve 1986;9:355-63.

35 Allen DE, Johnsson AG, Woolf AL. The intramuscular nerve endings in dystrophia myotonica-a biopsy study by vital staining and electron microscopy. J Anat 1969;10:1-26.

36 Fardeau M, Tomé FMS. Light and electron microscopic study of motor endplates in the adult and neonatal forms of dystrophia myotonica (preliminary results). From: Ontogenesis and Functional Mechanisms of Peripheral Synapses. INSERM, Symposium No 13, 1986:287-98. Amsterdam: Elsevier/North-Holland Biomedical Press.

37 Drachman DB, Fambrough DM. Are muscle fibers denervated in myotonic dystrophy? Arch Neurol 1976;33:485-8.

38 Lambert EH, Millikan CH, Eaton LM. Electrical stimulation in myotonia congenita. Am J Physiol 1952;17:741.

39 Pollock M, Dyck PJ. Peripheral nerve morphometry in myotonic dystrophy. Arch Neurol 1976;33:33-9. 